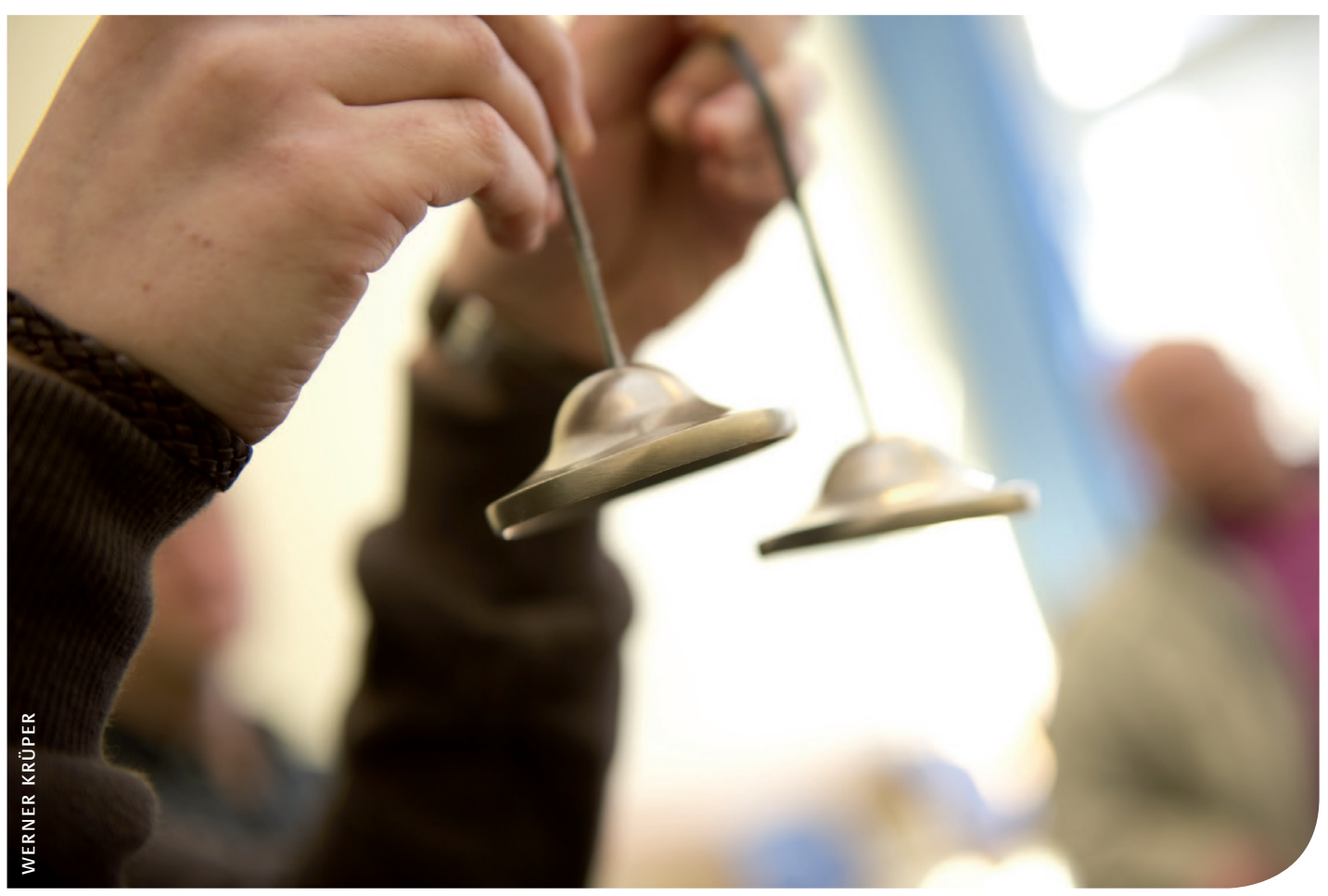

DEN TAG ACHTSAM BEENDEN

\title{
Die Abendrunde - „Behütet in die Nacht“
}

André Nienaber, Marie Boden

Rituale vereinfachen das Leben. Sie schaffen Vertrauen und strukturieren den Alltag. Die Station A2 der Klinik für Psychiatrie und Psychotherapie Bethel im Evangelischen Krankenhaus Bielefeld beschreitet diesen Weg seit zwei Jahren mit ihrer „Abendrunde“, die mittlerweile fester Bestandteil des Wochenprogramms ist.

\section{Einführung}

Eine Patientin der Station, eine 70-jährige Dame, die aufgrund ihrer manischdepressiven Erkrankung den schützenden Rahmen der Klinik benötigt, hat am Nachmittag einen heftigen Streit mit ihrer Tochter. Am Schluss des Besuchs fällt der Satz: „Dann wirst Du enterbt!“ Die Tochter verlässt wütend die Station, die Patientin schimpft über mangelnde Unterstützung seitens ihrer Tochter. Einige Stunden später zieht dieselbe Patientin zum Abschluss der Abendrunde [1] die „Gute Nacht-Karte ${ }^{\circledR \text { “ }}$ „Vergebung“. Sie blickt eine Weile auf die von ihr gezogene Karte, plötzlich beginnt sie zu weinen. Mitpatienten der Runde und der die Gruppe moderierende Pflegende sprechen ihr Trost zu.

Ein Patient, der mit der Diagnose einer paranoiden Schizophrenie auf der Station behandelt wird, übernimmt im Rahmen der Abendrunde das Vorlesen der Geschichte. Die ebenfalls an der Runde teilnehmenden Mitpatienten hören aufmerksam zu. In dem Abschlussgespräch für seine Behandlung gibt er an, dass diese Geschichte ihn sehr nachdenklich gestimmt habe.

Solche und ähnliche Begegnungen mit den Patientinnen und Patienten gibt es immer wieder, seit im Dezember 2009 auf Station A2 der Klinik für Psychiatrie und Psychotherapie Bethel im Evangelischen Krankenhaus Bielefeld (EvKB) die Abendrunde - zunächst noch als Projekt und mittlerweile als fester Bestandteil des wöchentlichen Programms - eingeführt wurde. Dabei handelt es sich bei der Abendrunde um eine pflegerische Gruppe, die sich in Anlehnung an Rakel und Lanzenberger [2] mit den Ressourcen des noch kranken Menschen befasst. $\mathrm{Zu}$ Beginn war die Abendrunde gedacht als ein freiwilliges Angebot für die Patientinnen und Patienten der Station in der Zeit von Montag bis Freitag. Ziel war und ist es, eine entspannte Begegnung im Krankenhausalltag zu ermöglichen und den Abend zu strukturieren. Mittlerweile ist die Abendrunde zu einem festen, ritualisierten Bestandteil des Wochenangebots geworden.

Rituale vereinfachen das Leben. Sie schaffen Vertrauen, sind verlässlich und strukturieren den Alltag. Jeder Mensch lebt unbewusst mit Ritualen. Ein eigener Rhythmus gehört zu den Grundbedürfnissen des Menschen. Insbesondere in schwierigen Lebensphasen sind Rituale hilfreich und wirken stabilisierend [3]. 


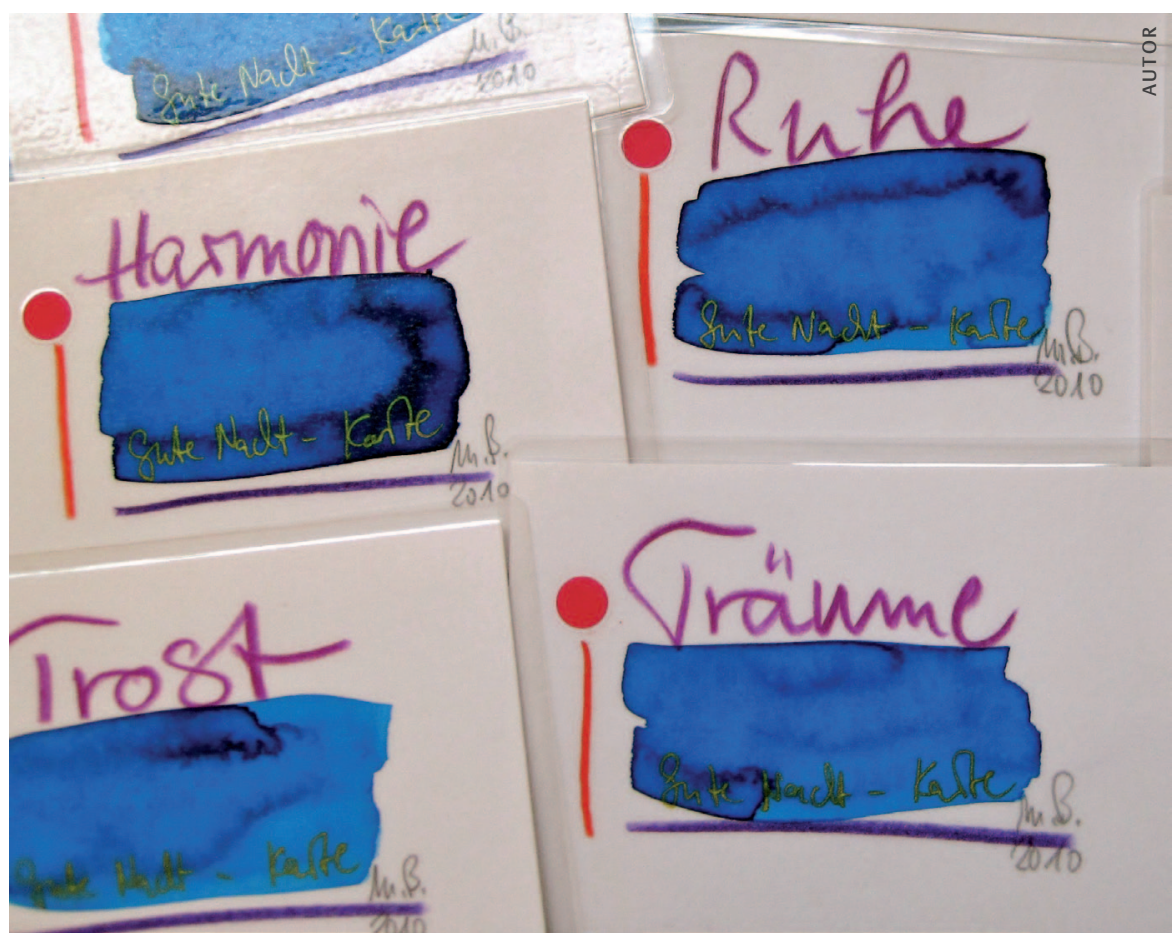

Abb. 2 Gute-Nacht Karten.

\section{Ziele der Abendrunde}

Ziel der Abendrunde ist zum einen die Schaffung eines „Ermöglichungsraumes“ [4] für eine Begegnung mit den Patientinnen und Patienten der Station, die sich in der Stabilisierungs- oder Transferphase ihrer Behandlung befinden. Dabei soll sie v.a. wertvolle Assistenz und Unterstützung [5] sein und die von Richter, Schwarze und Hahn [6] als zentrale Elemente der pflegerischen Beziehung identifizierten Ansatzpunkte aufgreifen. Dies sind:

- eine empathische und akzeptierende Grundhaltung (Einfühlen und Mitfühlen),

- die Förderung der Selbstbefähigung der Nutzerinnen und Nutzer und

- die Vermittlung von Hoffnung auf die persönliche Entwicklung [6].

Zum anderen hat die Abendrunde das Ziel des bewussten Wechsels vom Tag in den Abend bzw. die Nacht und unterstützt somit beim

\section{Im Sinne von Recovery rücken Klienten und professionelle} Helfer in der Abendrunde näher zusammen. Die Erlebnisse der Teilnehmenden und der Mitarbeitenden stehen im Mittelpunkt und ermöglichen eine Begegnung auf Augenhöhe.

„Slow-down“. Die Teilnehmenden sollen die Mühen des Tages hinter sich lassen und den Tag angenehm beenden. Dieses Ziel wird u.a. dadurch erreicht, dass gemeinsam auf den Tagesablauf zurückgeblickt wird, den Fokus auf ein positives Ereignis gerichtet. Stattfinden soll dies in einer angenehmen, ruhigen und harmonischen Atmosphäre (fast intim und familiär). Im Sinne von Recovery [7, 8] rücken Klienten und professionelle Helfer in der Abendrunde näher zusammen. Die Erlebnisse der Teilnehmenden und der Mitarbeitenden stehen im Mittelpunkt und ermöglichen eine Begegnung auf Augenhöhe.

Die Moderatoren nehmen die Rolle eines Wegbereiters ein, indem sie Offenheit und Authentizität vermitteln, und ihre eigenen Grenzen im Blick behalten. Daneben erfüllen sie die klassischen Moderatorenaufgaben wie Ausgleich und Integration. Sie vermitteln zwischen den Teilnehmenden und validieren jeden Wortbeitrag. Es geht v.a. um Wertschätzung, Sinnfindung und Alltagsbe- vidson [10] die Pflegenden dabei, sich ihrer eigenen Einstellungen bewusst zu werden und der Wichtigkeit ihres Verhaltens für die Gestaltung der professionellen Beziehung.

Wir gehen davon aus, dass auch von der Abendrunde als Gruppe eine Kohäsionskraft [11] ausgeht bzw. dass sich in der Gemeinschaft der Abendrunde unwillkürlich für die Teilnehmenden weitere therapeutische Hilfsmittel ergeben [3]. Zu benennen sind hier z.B. die Entstehung wichtiger und vertrauensvoller Beziehungen oder die Entwicklung von Solidarität. Christ und Hoffmann-Richter [12] bezeichnen diese Hilfsmittel auch als „das Handwerkliche“. Die Vermittlung von Hoffnung als „eine Haltung, eine Atmosphäre, eine Art und Weise, wie ich als Fachperson den betroffenen Menschen betrachte und wie ich ihm in der Folge begegne“ " [13] spielt eine wesentliche Rolle, z. B. im Sinne von Yalom [14] dadurch, dass Patienten erleben, wie sich Mitpatienten positiv entwickeln. Hedberg und Skärsäter [8] zufolge motiviert Hoffnung Menschen mit psychischen Erkrankungen zu Veränderungen und dazu, die Kontrolle über ihre Situation zurückzuerlangen.

\section{Hintergrund}

Patientinnen und Patienten, denen es besser geht, erhalten im Krankenhausalltag häufig weniger Aufmerksamkeit und $\mathrm{Zu}$ wendung durch Pflegende als die Patientinnen und Patienten, die sich in einer akuten Phase ihrer Erkrankung befinden. Dies trifft auch für die Patientinnen und Patienten im psychiatrischen Krankenhaus zu. Besonders auf einer fakultativ geschlossenen allgemeinpsychiatrischen Station wie der Station A2 - mit dem Schwerpunkt der Behandlung von Menschen mit akuten schizophrenen und schizoaffektiven Störungen erfordert der Alltag oftmals ein hohes Maß an Flexibilität: sowohl von den Mitarbeitenden als auch von den Patientinnen und Patienten wältigung im Sinne eines Gefühls des Gesehenwerdens und respektvollen Umgangs mit Blick auf die persönlichen Ressourcen [9]. Darüber hinaus unterstützen diese Werte im Sinne von Svedberg, Jormfeldt und Ar- selbst. Mit der Einführung der Abendrunde soll diesem Umstand Rechnung getragen werden, indem ein „Ermöglichungsraum für Begegnung und Teilhabe“ [4] geschaffen wird. 
Für die Patientengruppe, die sich in der Stabilisierungs- bzw. Transferphase ihrer Behandlung befindet, entsteht im Tagesablauf ein Zeitfenster, das sie für sich nutzen kann und gleichzeitig zur Tagesstrukturierung beiträgt. Nach und nach entwickelte sich ein ritualisierter Ablauf für die Durchführung der Abendrunde, welche folgende Elemente beinhaltet.

\section{Achtsamkeitsübung}

$\mathrm{Zu}$ Beginn jeder Abendrunde dient die Achtsamkeitsübung dazu, einen gemeinsamen Anfang zu ermöglichen und gemeinsam Stille zu erleben, innezuhalten, sich auf eine Sache zu konzentrieren. Die Dauer von einer Minute ist für alle Teilnehmenden gut aushaltbar. Der Klang der Zimbeln, der Beginn und Ende der Achtsamkeitsübung markiert, schafft eine Atmosphäre des Besonderen und betont den Augenblick. Im Anschluss an die Achtsamkeitsübung findet eine kurze Rückmeldung durch die Teilnehmenden statt. Mit ihrem Wortbeitrag integrieren sie sich in die Gruppe. Die aktive Teilnahme, das Zuhören, die Toleranz untereinander und das Erkennen anderer Blickwinkel werden unterstützt.

\section{Tagesrückblick}

Die Teilnehmenden blicken am Ende des Tages auf Erlebtes zurück. Einige nutzen den Tagesrückblick für die Reflexion ihrer Behandlung und berichten z.B. über Veränderungen und Fortschritte. Den Moderatoren bietet die Schilderung des Tagesablaufes die Möglichkeit zur positiven und wertschätzenden Rückmeldung. Auch kleine Erfolge können anerkannt werden, ebenso kann und soll nicht Gelungenes hier wertgeschätzt werden. Dieser Rückblick fördert das Reflektieren, das Erkennen positiver sowie nicht gelungener Erlebnisse im Alltag. Des Weiteren profitieren die Teilnehmenden voneinander, weil die Interaktion unterstützt wird. Freude, aber auch Leid kann an dieser Stelle geteilt werden. Gleichzeitig gibt es Raum, um zusammen zu lachen, aber auch zu weinen und Trost zu spenden oder zu empfangen.

Vorlesen

Das Vorlesen einer Geschichte ermöglicht Genuss, Trost, Stille und Konzentration. In der Rückmeldung berichten die Teilnehmenden, dass sie das Vorlesen einer Geschichte als kleines Geschenk erleben, Erinnerungen an die Kinderzeit werden geweckt. Die Texte und Geschichten können Rat geben, Zuflucht bieten, zum Innehalten einladen oder einfach nur Spaß machen. Bereits die alten Griechen nutzten das

sitiven Begriff, der den Tag abrundet und auf die Nacht vorbereitet.

Die Karten helfen, die Aktivitäten des Tages abzuschließen, sie rufen zum Innehalten auf, sie können Inhalt einer abendlichen Meditation sein. Manchmal tragen sie dazu bei, „herunterzufahren“ und zur Ruhe zu kommen, ein anderes Mal sind sie einfach nur da, um den Tag mit einem guten Wort zu beenden.

Ein positives Wort am Abend kann gelegentlich „kleine Wunder“ bewirken und für einen Augenblick den Blickwinkel ändern, Licht ins Dunkle bringen, Trost spenden oder zu einer dankbaren Haltung führen. Nicht zuletzt beenden die Gute-NachtKarten ${ }^{\circledR}$ auch die Abendrunde [15].

\section{Ausblick}

Mittlerweile blicken wir auf einen Zeitraum von zwei Jahren zurück, in dem sich die Abendrunde als fester Bestandteil im Wochenablauf der Station etabliert hat. Die Abendrunde wird insgesamt gut von den Patientinnen und Patienten angenommen und

Vorlesen zu therapeutischen Zwecken. In den USA wird es von Therapeuten bereits seit dem 19. Jahrhundert in Form der sogenannten Bibliotherapie auch für die Behandlung von Menschen mit psychischen Erkrankungen eingesetzt [14]. Im Anschluss an das Vorlesen kann sich eine Diskussion über die eben gehörte Geschichte ergeben. Alternativ kann das Vorlesen auch von einem anderen Teilnehmenden als dem Moderator übernommen werden.

\section{Gute-Nacht-Karten ${ }^{\circledR}$}

Der „Gute-Nacht-Karten ${ }^{\circledR}$ “-Satz besteht aus 22 Karten. Jede Karte enthält einen po- positiv bewertet. Eine Auswertung des ersten Jahres über die Anzahl der Teilnehmenden für den Zeitraum Januar bis Dezember 2010 hat ergeben, dass durchschnittlich fünf Personen an der Abendrunde teilgenommen haben. Im Durchschnitt findet sie an 14 Abenden im Monat statt. Dabei sind die Teilnehmenden zu zwei Dritteln weiblich.

Mehr und mehr übernehmen Teilnehmende von sich aus Elemente der Abendrunde, wie z.B. die Vorbereitung des Raumes, den Ausschank des für die Runde vorbereiteten Tees, das Vorlesen der Geschichte, das Verteilen der Gute-Nacht-Karten ${ }^{\circledR}$

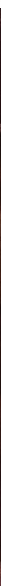




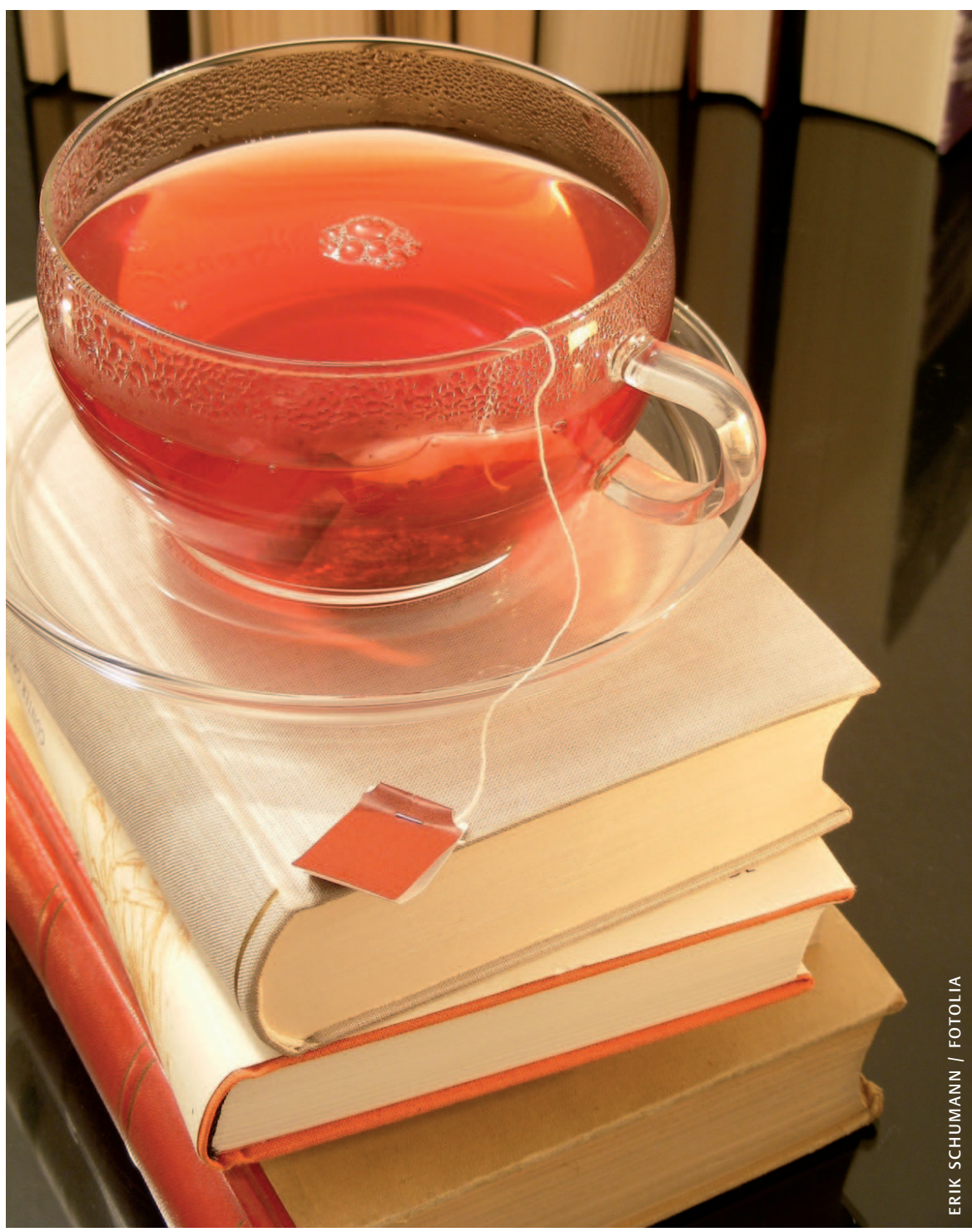

oder das Auspusten der Abendkerze. Auch für die Einhaltung des rituellen Ablaufs fühlen sich die teilnehmenden Patientinnen und Patienten zunehmend verantwortlich, indem sie die Moderatoren an den vorgesehenen Ablauf erinnern.

\section{Schlussfolgerungen}

Wir sind davon überzeugt, dass die Abendrunde einen großen Beitrag zu einer Begegnung auf Augenhöhe von Betroffenen und Professionellen leistet und in einfacher Weise einen an den vorhandenen Ressourcen orientierten Behandlungsansatz unterstützt. Die Abendrunde stellt dabei eine Möglichkeit dar, eine positive Einstellung gegenüber den Patientinnen und $\mathrm{Pa}$ tienten zu entwickeln - mit dem Ziel, sie aktiv in ihren eigenen Gesundungsprozess einzubeziehen [10].

\section{Glossar}

Kohäsionskraft. Kohäsionskraft bezeichnet nach Yalom „das gruppentherapeutische Gegenstück zur therapeutischen Beziehung in der Einzeltherapie“, die einen positiven Einfluss auf das Therapieergebnis hat.

\section{Literatur}

1 Boden, M.u. Nienaber, A. (2010). Abendrunde - Behütet in die Nacht. In: Hahn, S.; Schulz, M.; Schoppmann, S.; Abderhalden, C.; Stefan, H. \& Needham, I. (Hrsg.). „Depressivität und Suizidalität“ - Prävention - Früherkennung - Pflegeinterventionen - Selbsthilfe. Unterostendorf: Ibicura, 2010, S. 3-7

2 Rakel, T.u. Lanzenberger, A. (2001). Pflegetherapeutische Gruppen in der Psychiatrie. Planen - durchführen dokumentieren - bewerten. Stuttgart: Wissenschaftliche Verlagsgesellschaft.

3 Boden, M. u. Rolke, D. (2008). Krisen bewältigen, Stabilität erhalten, Veränderung ermöglichen. Ein Handbuch

zur Gruppenmoderation und zur Selbsthilfe. Psychosoziale Arbeitshilfen 25. Bonn: Psychiatrie-Verlag.

4 Schernus, R. (2010). Zur Ökonomisierung des Sozialen. Sozialpsychiatrische Informationen, 2, S. 7-14.

5 Mead, S. u. Copeland, M. E. (2000). What Recovery means to us: Consumers' Perspectives. Community Mental Health Journal, 36, S. 315-328.

6 Richter, Dirk; Schwarze, Thomas \& Hahn, Sabine (2010). Merkmale guter psychiatrischer Pflege und Betreuung. Psychiatrische Pflege Heute, 16, S.17-20.

7 Knuf, A. u. Bridler, S. (2008). Recovery konkret. Psychosoziale Umschau, 4, S. 26-29.

8 Repper, J. (2009). Recovery, Psychiatrie und Pflege. Zeitschrift für Pflegewissenschaft und psychische Gesundheit, 3, S. 1-11.

9 Hedberg, L. u. Skärsäter, I. (2009). The Importance of health for persons with psychiatric disabilities. Journal of Psychiatric and Mental Health Nursing, 16, S. 455-461. 10 Svedberg, P.; Jormfeldt, H. u. Arvidsson, B. (2003). Patients' conceptions of how health processes are promoted in mental health nursing. A qualitative study. Journal of Psychiatric and Mental Health Nursing, 10, S. 448-456. 11 Yalom, I.D. (2007). Theorie und Praxis der Gruppenpsychotherapie. Stuttgart: Klett-Cotta.

12 Christ, J.u. Hoffmann-Richter, U.. (1997). Therapie in der Gemeinschaft. Gruppenarbeit, Gruppentherapie und Gruppenpsychotherapie im psychiatrischen Alltag. Bonn: Psychiatrie-Verlag.

13 Knuf, A. (2004). Vom demoralisierenden Pessimismus zum vernünftigen Optimismus - Eine Annäherung an das Recovery-Konzept. Soziale Psychiatrie, 1, S. 38-41.

14 Merten, M. (2002). Nicht darauf vertrauen - nur hoffen. Deutsches Ärzteblatt, 12, S. 558-559.

15 Boden, M. (2010). Anleitung Gute Nacht-Karten [Unveröffentlichtes Skript]. Kontakt Marie Boden, E-Mail: Marie.Boden@evkb.de. Evangelisches Krankenhaus Bielefeld.

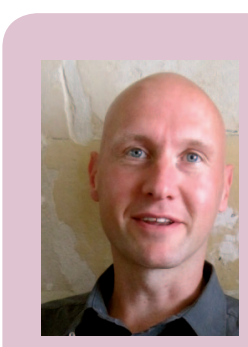

André Nienaber

Bachelor of Arts (B.A.), bis Juli 2010 Pflegeexperte für die Abteilung Allg. Psychiatrie, tätig als Pflegedienstleitung LWLKlinik Lengerich, Abt. für Allgemeine Psychiatrie und Psychotherapie, Abt. für Psychosomatische

Medizin und Psychotherapie,

Kontakt: andre.nienaber@wkp-Iwl.org

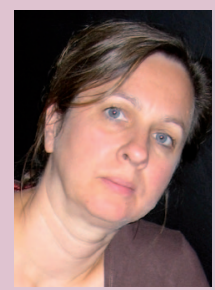

Marie Boden

Diplom-Designerin, Erzieherin. Seit 2003 Primary Nurse, seit 2009 DBT-Therapeutin für Sozial- und Pflegeberufe. Seit 2001 Aufbau und Konzeption der Stabilisierungsgruppe, 2008 Veröffentlichung der Arbeitshilfe „Krisen bewältigen, Stabilität erhalten, Veränderungen ermöglichen“. 2011 Veröffentlichung der Arbeitshilfe „Gefühle erkennen, annehmen und gut mit ihnen umgehen“.

\section{Kontaktadressen:}

Andre.Nienaber@evkb.de

Marie.Boden@evkb.de

\section{Bibliografie}

DOI 10.1055/s-0031-1301034

Psych Pflege 2012; 18: 47-50

(c) Georg Thieme Verlag KG

Stuttgart · New York · ISSN 0949-1619 